


\title{
ON THE DETERMINATION OF SETS BY THE SETS OF SUMS OF A CERTAIN ORDER
}

\author{
B. Gordon, A. S. Fraenkel and E. G. Straus
}

1. Introduction. Let $X=\left\{x_{1}, \cdots, x_{n}\right\}$ be a set of (not necessarily (iistinct) ${ }^{1}$ elements of a torsion free Abelian group. Define $P_{s}(X)=$ $\left\{x_{i_{1}}+x_{i_{2}}+\cdots+x_{i_{s}} \mid i_{1}<i_{2}<\cdots<i_{s}\right\}$. Thus $P_{s}(X)$ has $\left(\begin{array}{l}n \\ s\end{array}\right)$ (not necessarily distinct) elements. We introduce the equivalence relation $X \sim Y$ if and only if $P_{s}(X)=P_{s}(Y)$. Let $F_{s}(n)$ be the greatest number of sets $X$ which can fall into one equivalence class. Our purpose in this paper is to study conditions under which $F_{s}(n)>1$. Clearly $F_{s}(n)=\infty$ if $n \leqq s$ so that we may restrict our attention to $n>s$.

In [5] Selfridge and Straus studied this question, restricting attention to sets of elements of a field of characteristic 0 . In $\S 2$ we show that the numbers $F_{s}(n)$ remain the same even if we restrict ourselves to sets of positive integers. Thus the results in [5] remain valid in our case. These included a necessary condition for $F_{s}(n)>1$ and the proof that $F_{2}(n)>1$ (and hence $F_{n-2}(n)>1$ ) if and only if $n$ is a power of 2 . Also $F_{s}(2 s)>1$.

In $\S 3$ we give a simpler form of the necessary condition in [5].

In $\S 4$ we examine this necessary condition and prove that for $s>2$ we have $F_{s}(n)=1$ for all but a finite number of $n$. This was conjectured in [5]. The method seems to be of independent interest since it can be applied to a class of Diophantine equations in two unknowns which are algebraic in one and exponential in the other variable.

In $\S 5$ we apply the methods of [5] to show that $F_{2}(8)=3, F_{2}(16) \leqq$ $3, F_{3}(6) \leqq 6$ and $F_{4}(12) \leqq 2$.

The fact that $F_{2}(8)=3$ disproves the conjecture $F_{2}(n) \leqq 2$ made in [5]. Except for the corresponding result $F_{6}(8)=3$ we have not found another nontrivial case in which we can prove $F_{s}(n)>2$.

In the final section we adapt a method of Lambek and Moser [3] to the case $s=2$ and get a partial characterization of those sets which are equivalent to other sets.

2. Reduction to sets of integers. In this section we demonstrate that there exist $F_{s}(n)$ distinct equivalent sets of positive integers so that in any effort to evaluate $F_{s}(n)$ we may restrict our attention to sets of integers.

Received March 29, 1961. The work of the third author was supported in part by the National Science Foundation.

1 Throughout this paper we use the word "set" to mean "set with multiplicities" in the sense in which one speaks of the set of zeros of a polynomial. 
Let $N=F_{s}(n)$ and let $X_{1}=\left\{x_{11}, \cdots, x_{n 1}\right\}, \cdots, X_{N}=\left\{x_{1 N}, \cdots, x_{n N}\right\}$ be the members of a maximal equivalence class. Since the $x_{i j}$ form of a finite set of elements of a torsion-free Abelian group, they generate a group with basis $y_{1}, \cdots, y_{m}$ over the integers (see e.g. [2], Theorem 6). In other words every $x_{i j}$ can be represented as an $m$-vector, $x_{i j}=$ $\left(a_{i j}^{1}, \cdots, a_{i j}^{m}\right)$ with integral components. The addition of a fixed $m$-vector with sufficiently large components to all $x_{i j}$ does not effect the equivalence of the $X_{j}$ so that we may assume that every $a_{i j}^{k}$ is non-negative. Now let $A$ be an integer with $A>s \max a_{i j}^{k}$ and associate to each $x_{i j}$ the number

$$
y_{i j}=a_{i j}^{1}+a_{i j}^{2} A+\cdots+a_{i j}^{m} A^{m-1} .
$$

It is now clear that two sums of $s$ or fewer $y_{i j}$ are same if and only if the corresponding sums of $x_{i j}$ are the same. In other words the sets of integers $Y_{j}=\left\{y_{1 j}, \cdots, y_{n j}\right\}(j=1, \cdots, N)$ form an equivalence class with $N=F_{s}(n)$ distinct members.

\section{Simplification of the necessary conditions for $F_{s}(n)>1$.}

In this section we show that the Diophantine equation $f(n, k)=0$ of [5] can be writen in the form

$$
\left(\begin{array}{c}
n \\
s-1
\end{array}\right)-\left(\begin{array}{c}
n \\
s-2
\end{array}\right) 2^{k-1}+\left(\begin{array}{c}
n \\
s-3
\end{array}\right) 3^{k-1}-\cdots+(-1)^{s-1} s^{k-1}=0 .
$$

To see this we start with the expression given in [5], namely

$$
f(n, k)=\frac{1}{s} \sum_{P}(-1)^{s t} n^{t-1} \sum_{i=1}^{r} a_{i} i^{k},
$$

where $P$ runs through all permutations on $s$ letters, $a_{i}$ is the number of cycles of length $i$ in $P$, and $t=\Sigma a_{\imath}$ is the total number of cycles in $P$. Changing the order of summation we get

$$
f(n, k)=\sum_{i} i^{k-1}(-1)^{s-1} \frac{i}{s} \sum_{t=1}^{s-i}(-n)^{t-1} N_{i t},
$$

where $N_{i t}$ is the number of permutations $P$ which contain exactly $t$ cycles, including at least one $i$-cycle. Since there are $\left(\begin{array}{l}s \\ i\end{array}\right)(i-1)$ ! choices of the one $i$-cycle, and $[(s-i) ! /(t-1) !] \sum_{\Sigma c_{j}=s-i} 1 /\left(c_{1} c_{2} \cdots c_{t-1}\right)$ choices of the other cycles of length $c_{1}, \cdots, c_{t-1}$, we have

$$
\begin{aligned}
f(n, k)= & \sum_{i} i^{k-1} \frac{(-1)^{s-1} i}{s} \frac{s !}{(s-i) ! i !}(i-1) !(s-i) ! \\
& \cdot \sum_{t=1}^{s-i} \frac{(-n)^{t-1}}{(t-1) !} \sum_{\Sigma c_{j=s-i}} \frac{1}{c_{1} c_{2} \cdots c_{t-1}} \\
= & \sum_{i} i^{k-1}(-1)^{s-1}(s-1) ! \sum_{t=1}^{s-i} \frac{(-n)^{t-1}}{(t-1) !} \sum_{\Sigma c_{j=s-i}} \frac{1}{c_{1} c_{2} \cdots c_{t-1}}
\end{aligned}
$$


Now if $|x|<1$, we have

$$
\begin{aligned}
-\log (1-x) & =\sum_{c=1}^{\infty} \frac{x^{c}}{c}, \\
(-1)^{v} \log ^{v}(1-x) & =\sum_{w=v}^{\infty} x^{w} \sum_{2 c_{j}=w} \frac{1}{c_{1} c_{2} \cdots c_{v}} .
\end{aligned}
$$

Multiplying by $(-n)^{n} / v$ ! and summing over $v$ we obtain

$$
\begin{aligned}
(1-x)^{n}=e^{n \log (1-x)} & =\sum_{v=0}^{\infty} \frac{n^{v} \log ^{v}(1-x)}{v !} \\
& =\sum_{p=0}^{\infty} x^{p} \sum_{v=0}^{p} \frac{(-n)^{v}}{v !} \sum_{c_{1}+\cdots+c_{v}=p} \frac{1}{c_{1} c_{2} \cdots c_{v}}
\end{aligned}
$$

from which we deduce that

$$
(-1)^{w}\left(\begin{array}{c}
n \\
w
\end{array}\right)=\sum_{v=0}^{w} \frac{(-n)^{v}}{v !} \sum_{c_{1}+\cdots+c_{v}=w} \frac{1}{c_{1} c_{2} \cdots c_{v}} .
$$

Putting $v=t-1, w=s-i$, we obtain

$$
\begin{aligned}
f(n, k) & =\sum_{i} i^{k-1}(-1)^{s-1}(s-1) !(-1)^{s-i}\left(\begin{array}{c}
n \\
s-i
\end{array}\right) \\
& =\sum_{i} i^{k-1}(s-1) !(-1)^{i-1}\left(\begin{array}{c}
n \\
s-i
\end{array}\right) .
\end{aligned}
$$

Therefore the equation $f(n, k)=0$ is equivalent to $\sum_{i} i^{k-1}(-1)^{i-1}\left(\begin{array}{c}n \\ s-i\end{array}\right)=0$.

4. Proof that for $s>2$ we have $F_{s}(n)=1$ for all but a finite number of $n$.

LEMma. For large values of $k$ the equation (1) has $s-1$ real roots $n=n_{1}, \cdots, n_{s-1}$ where

$$
n_{j}=(s-j)(1+1 / j)^{k-1}+O\left((1+1 / j)^{\delta k}\right), \quad \delta<1 .
$$

Proof. Divide the left side of (1) by $(-1)^{j-1}\left(\begin{array}{c}n \\ s-j-1\end{array}\right) j^{k-1}$ and then consider its behavior in the neighborhood $N_{j}$ of $n=n_{l}^{*}=(s-j)(1+1 / j)^{k-1}$ say $N_{j}=\left\{n \mid n_{j}^{*} / 2 \leqq n \leqq 2 n_{j}^{*}\right\}$. We have

$$
\left(\begin{array}{c}
n \\
s-i
\end{array}\right) i^{k-1} /\left(\begin{array}{c}
n \\
s-j-1
\end{array}\right) j^{k-1}<c_{1} n^{j-i+1}(i / j)^{k}<c_{2}\left(i(j+1)^{j-i+1} / j^{i-j+2}\right)^{k}=c_{2} l_{\imath j}^{k} .
$$

It remains to show that $l_{i j}<1+1 / j$ for all $i \leqq i<j$ and all $j+1<i \leqq$ $s-1$. For $i<j$ this leads to

$$
1+\frac{1}{j}<\left(1-\frac{j-i}{j}\right)^{1 /(i-j)}=1+\frac{1}{j}+\cdots
$$


and for $i>j+1$ to

$$
i / j<(1+1 / j)^{i-j}=1+(i-j) / j+\cdots .
$$

Thus, if we set

$$
\delta=\max _{\substack{1 \leq i<j \\ j+1<i<8}}\{j-i+1+\log (i / j) / \log (1+1 / j)\}
$$

Then $\delta<1$ and (1) becomes

$$
(n-s+j+1) /(s-j)-(1+1 / j)^{k-1}+O\left((1+1 / j)^{\delta k}\right)=0
$$

for $n \in N_{j}$. Thus (1) must have a root in $N_{j}$ and according to (3) this is the real root given in (2).

TheoRem. If $s>2$ then there is only a finite number of $n$ for which $F_{s}(n)>1$.

Proof. If the Diophantine equation (1) has solutions for arbitrarily large $k$ then by the Lemma the solutions are of the form

$$
n=(s-j)(1+1 / j)^{k-1}+O\left(n^{\delta}\right),
$$

where $1 \leqq j \leqq s-1$ and $\delta<1$.

On the other hand all solutions of (1) satisfy $n \mid(s-1)$ ! $s^{k-1}$ so that all prime factors of $n$ are less than or equal to $s$. The same holds for the prime factors which occur in the numerator and denominator of $(s-j)(1+1 / j)^{k-1}$.

Now according to a Theorem of Ridout [4] for any $\varepsilon>0$ there is at most a finite number of integers $p, q$ whose prime divisors belong to fixed finite sets and which satisfy $0<|1-p / q|<1 / q^{\varepsilon}$; or, equivalently

$$
0<|q-p|<q^{1-\varepsilon} \text {. }
$$

But

$$
\left|n j^{k-1}-(s-j)(j+1)^{k-1}\right|<c j^{k-1} n^{\delta}<c_{1}\left(n j^{k-1}\right)^{1-\varepsilon} .
$$

for some $\varepsilon>0$, so that if there is an infinite number of solutions we must have $n j^{k-1}=(s-j)(j+1)^{k-1}$ infinitely often. For large $k$, this implies $j=1$ and $n=(s-1) \cdot 2^{k-1}$. For $s=2$ this does indeed give an infinite family of solutions, but for $s>2$ we see that for $n=(s-1) \cdot 2^{k-1}$

$$
\begin{aligned}
& \left(\begin{array}{c}
n \\
s-1
\end{array}\right)-\left(\begin{array}{c}
n \\
s-2
\end{array}\right) 2^{k-1}=O\left(2^{(s-2) k}\right) \\
& \left(\begin{array}{c}
n \\
s-j
\end{array}\right) j^{k-1}=O\left(2^{(s-j) k} j^{k}\right)
\end{aligned}
$$

so that the third term in (1) dominates the sum of the first two terms 
as well as all the subsequent terms and the equation cannot hold for large $k$.

Using a method of Davenport and Roth [1] we could obtain an upper bound on the number of $n$ for which $F_{s}(n)>1$, but this bound would probably be far from best possible.

5. Special cases. As in [5] we put $S_{k}=\sum_{i=1}^{n} x_{i}^{k}$ and $\Sigma_{k}=$ $\Sigma\left(x_{i_{1}}+\cdots+x_{i_{s}}\right)^{k}$, the summation being extended over all indices $i_{1}, \cdots, i_{s}$ with $1 \leqq i_{1}<i_{2}<\cdots<i_{s} \leqq n$. Then each $\Sigma_{k}$ can be expressed as a polynomial in $S_{1}, \cdots, S_{k}$. Since all sets $X$ of an equivalence class give rise to the same $\Sigma_{k}$ 's, and since the elements of $X$ are uniquely determined by $S_{1}, \cdots, S_{n}$, we can obtain an upper bound for $F_{s}(n)$ by estimating the number of different $n$-tuples $\left(S_{1}, \cdots, S_{n}\right)$ corresponding to a given set of $\Sigma$ 's. Since $\Sigma_{1}=\left(\begin{array}{c}n-1 \\ s-1\end{array}\right) S_{1}$ we see that all members of an equivalence class have the same $S_{1}$. We can assume without loss of generality that $S_{1}=0$.

The case $s=2, n=8$.

In this case there are $28 \Sigma$ 's, and the first 12 of them are given by the following expressions (for $S_{1}=0$ )

(1) $\quad \Sigma_{1}=0$

(2) $\quad \Sigma_{2}=6 S_{2}$

(3) $\quad \Sigma_{3}=4 S_{3}$

(4) $\quad \Sigma_{4}=3 S_{2}^{2}$

(5) $\quad \Sigma_{5}=-8 S_{5}+10 S_{2} S_{3}$

(6) $\quad \Sigma_{6}=-24 S_{6}+15_{2} S_{4}+10 S_{3}^{2}$

( 7 ) $\quad \Sigma_{7}=-56 S_{7}+21 S_{2} S_{5}+35 S_{3} S_{4}$

(8) $\quad \Sigma_{8}=-120 S_{8}+28 S_{2} S_{6}+56 S_{3} S_{5}+35 S_{4}^{2}$

( 9 ) $\quad \Sigma_{9}=-248 S_{9}+36 S_{2} S_{7}+84 S_{3} S_{6}+126 S_{4} S_{5}$

(10) $\quad \Sigma_{10}=-504 S_{10}+45 S_{2} S_{8}+120 S_{3} S_{7}+210 S_{4} S_{6}+126 S_{5}^{2}$

(11) $\quad \Sigma_{11}=-1016 S_{11}+55 S_{2} S_{9}+165 S_{3} S_{8}+330 S_{4} S_{7}+462 S_{5} S_{6}$

(12) $\quad \Sigma_{12}=-2040 S_{12}+66 S_{2} S_{10}+220 S_{3} S_{9}+495 S_{4} S_{8}+792 S_{5} S_{7}+462 S_{6}^{2}$.

Equations (2), (3), and (5) show that $S_{2}, S_{3}$, and $S_{5}$ are uniquely determined by the $\Sigma$ 's. Furthermore (6), (7), and (8) imply that $S_{6}, S_{7}$, and $S_{8}$ are uniquely determined by the $\Sigma$ 's once $S_{4}$ is known. So to prove $F_{2}(8) \leqq 3$, it is sufficient to show that corresponding to a given set of $\Sigma$ 's, there can be at most 3 values of $S_{4}$. Now $S_{9}, S_{10}, S_{11}$, and $S_{12}$ can be expressed in terms of $S_{1}, \cdots, S_{8}$ using the theory of symmetric functions. Since these in turn can be expressed in terms of $S_{4}$ and the $\Sigma$ 's, equations (9), (10), (11), and (12) give us four equations involving $S_{4}$ and the $\Sigma$ 's. Now (9) is linear in $S_{4},(10)$ and (11) are quadratic in $S_{4}$, while 
(12) is cubic in $S_{4}$. We shall show that the coefficient of $S_{4}^{3}$ in (12) is not zero, which implies that $S_{4}$ can have at most 3 values. Then, in order that it actually can have 3 values, we must have the coefficients of $S_{4}$ in (9), (10), (11) and the coefficients of $S_{4}^{2}$ in (10), (11) equal to 0. This gives considerable information on the structure of the 3 -member equivalence classes.

First we compute the coefficient of $S_{4}^{2}$ in equation (11). It arises only from the terms $-1016 S_{11}, 165 S_{3} S_{8}$ and $330 S_{4} S_{7}$. The last term contributes $330\left((35 / 56) S_{3}\right) S_{4}^{2}=(825 / 4) S_{3} S_{4}^{2}$, making use of (7). To compute the contribution of $-1016 S_{11}$ we use the relation from the theory of symmetric functions

$$
0=\frac{1}{11} S_{11}-\frac{1}{18} S_{2} S_{9}-\frac{1}{24} S_{3} S_{8}+\frac{1}{96} S_{3} S_{4}^{2}-\frac{1}{28} S_{4} S_{7}+\cdots .
$$

This, combined with equations (7) and (8) gives

$$
\begin{aligned}
S_{11} & =\frac{11}{24} S_{3}\left(\frac{35}{120} S_{4}^{2}\right)+\frac{11}{28}\left(\frac{35}{56} S_{3} S_{4}\right) S_{4}-\frac{11}{96} S_{3} S_{4}^{2}+\cdots \\
& =\frac{979}{96.35} S_{3} S_{4}^{2}+\cdots .
\end{aligned}
$$

From (8), the term $165 S_{3} S_{8}$ contributes $165 \cdot(35 / 120) S_{3} S_{4}^{2}$. Hence the coefficient of $S_{4}^{2}$ in equation (11) is

$$
\left(-1016 \cdot \frac{979}{96.35}+\frac{825}{4}+\frac{165.35}{120}\right) S_{3}
$$

where the number in parentheses is $\neq 0$. Thus in order for an equivalence class to contain 3 members, we must have $S_{3}=0$. Next consider the coefficient of $S_{4}$ in equation (9) (supposing from now on that $S_{3}=0$ ). It arises from the terms $-248 S_{9}$ and $126 S_{4} S_{5}$. But

$$
0=\frac{1}{9} S_{9}-\frac{1}{20} S_{5} S_{4}+\cdots,
$$

from which $S_{9}=(9 / 20) S_{5} S_{4}+\cdots$. So the coefficient of $S_{4}$ is

$$
-248\left(\frac{9}{20} S_{5}\right)+126 S_{5}=\frac{72}{5} S_{5} .
$$

Hence in order to have more than one member in such an equivalence class we must have $S_{5}=0$. Next consider the coefficient of $S_{4}$ in equation (11) (supposing $S_{3}=S_{5}=0$ ). It arises from $-1016 S_{11}$ and from $330 S_{4} S_{7}$. Since $0=(1 / 11) S_{11}-(1 / 28) S_{4} S_{7}+\cdots$ the coefficient is

$$
-1016\left(\frac{11}{28}\right) S_{7}+330 S_{7}=\frac{-584}{7} S_{7} .
$$


Hence we must have $S_{7}=0$ in order to have 3 sets in the same equivalence class. Finally the coefficient of $S_{4}^{3}$ in equations (12) arises from $-2040 S_{12}$ and from $495 S_{4} S_{8}$. Using the relation

$$
0=\frac{1}{12} S_{12}-\frac{1}{32} S_{4} S_{8}+\frac{1}{6 \cdot 64} S_{4}^{3}-\cdots
$$

and (8), we obtain a coefficient of

$$
(-2040)\left(\frac{12}{32}\right)\left(\frac{35}{120}\right)-2040\left(\frac{-12}{6 \cdot 64}\right)+495\left(\frac{35}{120}\right) \neq 0,
$$

which completes the proof that $F_{2}(8) \leqq 3$. Moreover we see from the proof that if $X, Y, Z$ form a 3 -member equivalence class (with $S_{1}=0$ ), then $X, Y, Z$ all have $S_{k}=0$ for $k$ odd, and hence each consists of 4 members and their negatives. In addition, there can be only one such equivalence class having a given value for $\Sigma_{6}$ and 3 given values for $S_{4}$. For the three given values of $S_{4}$ determine the coefficients of the cubic equation (12), and hence determine $\Sigma_{2}, \Sigma_{8}$, and $\Sigma_{13}$. But then all other $\Sigma$ 's are determined from these. Now if $a, b, c, d$ are any 4 numbers, then the sets $X=X_{1} \cup-X_{1}, Y=Y_{1} \cup-Y_{1}$, and $Z=Z_{1} \cup-Z_{1}$, where $X_{1}=\{a, b, c, d\}, \quad Y_{1}=\left\{\frac{1}{2}(-a+b+c+d), \frac{1}{2}(a-b+c+d), \frac{1}{2}(a+b-c+d)\right.$, $\left.\frac{1}{2}(a+b+c-d)\right\}$, and $Z_{1}=\left\{\frac{1}{2}(a+b+c+d), \frac{1}{2}(a+b-c-d), \frac{1}{2}(a-b+c-d)\right.$, $\left.\frac{1}{2}(a-b-c+d)\right\}$ are all equivalent. Furthermore if any 4 (complex) numbers $\Sigma_{6}, S_{4}^{\prime}, S_{4}^{\prime \prime}, S_{4}^{\prime \prime \prime}$ are given, it is possible to choose $a, b, c, d$ so that $\Sigma_{6}(X)=\Sigma_{6}(Y)=\Sigma_{6}(Z)=\Sigma_{6}, S_{4}(X) S_{4}^{\prime}, S_{4}(Y)=S_{*}^{\prime \prime}, S_{4}(Z)=S_{+}^{\prime \prime \prime}$. Indeed, it is easy to see that the prescribed conditions merely determine the symmetric functions of $a, b, c, d$, and of course one can always find complex $a, b, c, d$ for which these have preassigned values. It follows that the sets $X, Y, Z$ give a parametric representation of all 3 -member equivalence classes (with $S_{1}=0$ ).

Other values of $F_{s}(n)$. A similar treatment can be given for the other values of $F_{s}(n)$ mentioned in the introduction. We will omit the details and merely sketch the general method in these cases. If $s=2$, $n=4$, the first $S_{k}$ not uniquely determined by the $\Sigma$ 's is $S_{3}$, and all other $S_{k}$ are determined by $S_{3}$ and the $\Sigma$ 's. The equation for $\Sigma_{6}$ then becomes a quadratic equation in $S_{3}$ and the coefficient of $S_{3}^{2}$ in this equation is not 0 . Hence, corresponding to a given set of $\Sigma$ 's there can be at most 2 values of $S_{3}$, and accordingly at most 2 sets $X$ and $Y$. Thus $F_{2}(4) \leqq 2$. An argument similar to that given above shows that $F_{2}(4)=$ 2 and that all 2-member equivalence classes are given by $X=\{a, b, c, d\}$, $\left.Y=\frac{1}{2}(-a+b+c+d), \frac{1}{2}(a-b+c+d), \frac{1}{2}(a+b-c+d), \frac{1}{2}(a+b+c-d)\right\}$. In the case $s=2, n=16$, we find that $S_{5}$ is the first $S_{k}$ not uniquely determined by the $\Sigma$ 's, and that all other $S_{k}$ are uniquely determined by $S_{5}$ and the $\Sigma$ 's. The equation for $\Sigma_{17}$ gives a cubic equation for $S_{5}$, 
the coefficient of $S_{5}$ being a nonzero multiple of $S_{2}$. By $\S 2$ we can assume that the sets $X$ are real, and hence $S_{2}>0$. This proves $F_{2}(16) \leqq$ 3. On the other hand $F_{2}(16) \geqq 2$ as was shown in [4]. We do not know at present whether $F_{2}(16)=2$ or 3 . This type of reasoning can probably be made to yield the estimate $F_{2}\left(2^{k}\right) \leqq \alpha$, where $\alpha$ is the least integer such that $(k+1) \alpha>2^{k}$; however, this seems to be far from the best possible result.

For $s=4, n=12$ the first $S_{k}$ not uniquely determined by the $\Sigma$ 's is $S_{6}$, and all other $S_{k}$ are uniquely determined once $S_{6}$ is known. The equation for $\Sigma_{14}$ gives a quadratic equation for $S_{6}$, the coefficient of $S_{6}^{2}$ being a nonzero multiple of $S_{2}$. Hence $F_{4}(12) \leqq 2$. We do not know whether $F_{4}(12)=1$ or 2 .

Finally, if $s=3, n=6$, then the equations for the $\Sigma$ 's in terms of the $S$ 's show that $S_{2}$ and $S_{4}$ are uniquely determined by the $\Sigma$ 's, while $S_{6}$ is determined by the $\Sigma$ 's and by $S_{3}$. The equations for $\Sigma_{8}$ contains a term in $S_{3} S_{5}$ with nonvanishing coefficient. Hence it can be used to write $S_{5}=\left(\alpha S_{3}^{2}+\beta\right) / S_{3}$, where $\alpha, \beta$ depend on the $\Sigma$ 's.

Then the expression for $\Sigma_{12}$ yields a sextic equation for $S_{3}$ and the coefficient of $S_{3}^{6}$ is nonzero. Hence $2 \leqq F_{3}(6) \leqq 6$.

6. Generating functions for the case $s=2$. In this section we use a method suggested by Lambek and Moser [2] to obtain some results on equivalent sets in the case $s=2$.

Suppose $A=\left\{a_{1}, \cdots, a_{n}\right\}$ (where $0=a_{1} \leqq a_{2} \leqq \cdots \leqq a_{n}$ ) and $B=$ $\left\{b_{1}, \cdots, b_{n}\right\}$ (with $0 \leqq b_{1} \leqq \cdots \leqq b_{n}$ ) are equivalent sets of nonnegative integers. Construct the generating polynomials $f(x)=\Sigma x^{a_{i}}, g(x)=\Sigma x^{b_{i}}$. Then the generating polynomial for the set of sums is $\frac{1}{2}\left(f^{2}(x)-f\left(x^{2}\right)\right)=$ $\frac{1}{2}\left(g^{2}(x)-g\left(x^{2}\right)\right)$. Hence $f^{2}(x)-g^{2}(x)=f\left(x^{2}\right)-g\left(x^{2}\right)$. Let $F=f+g, G=$ $f-g$; then $F(x) G(x)=G\left(x^{2}\right)$, so that $G(x) \mid G\left(x^{2}\right)$. This is possible only if every zero of $G$ has a square which is itself a zero of $G$, in other words only if

$$
G(x)=c x^{\alpha} \prod_{i} \varphi_{i}(x),
$$

where the $\varphi_{i}$ are cyclotomic polynomials. We can write this, in the customary way, as

$$
G(x)=c x^{\alpha} \Pi\left(1-x^{\beta_{i}}\right) / \Pi\left(1-x^{\gamma}\right)
$$

where the $\beta_{i}$ and $\gamma_{j}$ are positive integers, and hence

$$
F(x)=\frac{G\left(x^{2}\right)}{G(x)}=x^{\alpha} \Pi\left(1+x^{\beta_{i}}\right) / \Pi\left(1+x^{\gamma_{j}}\right) .
$$

Since $F(1)=2 n$ is a power of 2 we have here a new and simple proof of the fact that $F_{2}(n)>1$ only when $n$ is a power of 2 . 
The problem of finding equivalent sets of integers now reduces to that of determining the $\beta_{i}$ and $\gamma_{j}$ (we must clearly set $\alpha=0$ ) for which the polynomials $F$ and $G$ have nonnegative coefficients. This makes $|c|=$ $|G(0)| \leqq F(0)=1$ in (13) necessary so that $c=1$. We certainly get nonnegative coefficients if there are no denominators (no $\gamma_{j}$ ) which proves $F_{2}\left(2^{k}\right)>1$ and permits a simple construction of equivalent classes of order $2^{k}$ :

Given $k+1$ numbers $\alpha_{0}, \cdots, \alpha_{k}$ let $X$ be the set of sums of an even number of $\alpha$ 's and $Y$ the set of sums of an odd number of $\alpha$ 's. Clearly $P_{2}(X)=P_{2}(Y)$. These are the sets which were obtained in [5].

However there do exist cases in which the $\gamma_{j}$ are not absent, for example

$$
G(x)=(1-x)^{4}\left(1-x^{2}\right)^{2}\left(1-x^{3}\right)^{3}\left(1-x^{6}\right)^{3}\left(1-x^{12}\right) /\left(1-x^{4}\right) .
$$

This cyclotomic polynomial leads to the following two sets $A$ and $B$ with $2^{11}$ elements each:

$\begin{array}{lllllllllllllllllllllll}\text { element } & 0 & 1 & 2 & 3 & 4 & 5 & 6 & 7 & 8 & 9 & 10 & 11 & 12 & 13 & 14 & 15 & 16 & 17 & 18 & 19 & 20 & 21\end{array}$ multiplicity in $A \begin{array}{lllllllllllllllllllllll} & 0 & 0 & 6 & 8 & 14 & 10 & 19 & 41 & 26 & 29 & 35 & 78 & 69 & 37 & 58 & 104 & 114 & 22 & 56 & 129 & 120 & 48\end{array}$

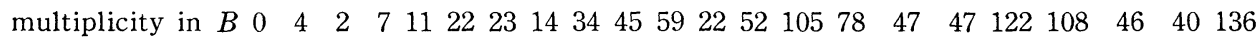

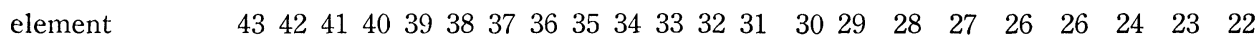

The symmetry in the multiplicities is typical since the cyclotomic polynomials are reciprocal. We have no example with non-trivial denominators in (14) which leads to two sets without multiple elements.

A complete characterization of the possible functions $F, G$ seems therefore difficult.

The fact that $F_{2}(8)=3$ in the notation of the introduction and the characterization of the classes containing three equivalent sets can now be understood from this point of view by noting that $f$ need not determine $F$ uniquely. Namely if we write $F=\left(1+x^{\alpha_{1}}\right)\left(1+x^{\alpha_{2}}\right)\left(1+x^{\alpha_{3}}\right)\left(1+x^{\alpha_{4}}\right)$ and $F^{*}=\left(1+x^{\beta_{1}}\right)\left(1+x^{\beta_{2}}\right)\left(1+x^{\beta_{3}}\right)\left(1+x^{\beta_{4}}\right)$ then $F$ and $F^{*}$ give rise to the same $f$ whenever the set of sums of an even number of $\alpha$ 's is the same as the set of sums of an even number of $\beta$ 's. In other words, whenever $\beta_{i}=\frac{1}{2}\left(\alpha_{1}+\alpha_{2}+\alpha_{3}+\alpha_{4}\right)-\alpha_{i}$ (after suitable reordering). The generating functions $f, g_{1}=F-f$ and $g_{2}=F^{*}-f$ then describe the three equivalent sets given in $\S 5$.

The question whether $F_{2}(n) \leqq 2$ for $n>8$ reduces to that of whether two different $F(x)$ and $F^{*}(x)$ can give rise to the same $f$ when $F(1)>16$.

\section{REFERENCES}

1. H. Davenport and K. F. Roth, Rational approximations to algebraic numbers, Mathematika, 2 (1955), 160-167.

2. I. Kaplansky, Abelian Groups, Michigan University Press. 
3. J. I.ambek and L. Moser, On some two way classifications of integers, Can. Math. Bull., 2 (1959), 85-89.

4. D. Ridout, Rational approximations to algebraic numbers, Mathematika, 4 (1957), 125131.

5. L. J. Selfridge and E. G. Straus, On the determination of numbers by their sums of a fixed order, Pacific J. Math., 8 (1958), 847-856.

UNIVERSITY OF CALIFORNIA,

LOS ANGELES 


\section{PACIFIC JOURNAL OF MATHEMATICS}

\section{EDITORS}

Ralph S. Phillips

Stanford University

Stanford, California

M. G. Arsove

University of Washington

Seattle 5, Washington
A. L. Whiteman

University of Southern Californla

Los Angeles 7, California

Lowell J. Paige

University of California

Los Angeles 24, California

\section{ASSOCIATE EDITORS}

E. F. BECKENBACH

D. DERRY

H. L. ROYDEN

E. G. STRAUS

T. M. CHERRY

M. OHTSUKA

E. SPANIER

F. WOLF

\section{SUPPORTING INSTITUTIONS}

UNIVERSITY OF BRITISH COLUMBIA

STANFORD UNIVERSITY

CALIFORNIA INSTITUTE OF TECHNOLOGY

UNIVERSITY OF CALIFORNIA

MONTANA STATE UNIVERSITY

UNIVERSITY OF TOKYO

UNIVERSITY OF UTAH

UNIVERSITY OF NEVADA

NEW MEXICO STATE UNIVERSITY

OREGON STATE UNIVERSITY

UNIVERSITY OF OREGON

OSAKA UNIVERSITY

WASHINGTON STATE UNIVERSITY

UNIVERSITY OF WASHINGTON

UNIVERSITY OF SOUTHERN CALIFORNIA

AMERICAN MATHEMATICAL SOCIETY CALIFORNIA RESEARCH CORPORATION SPACE TECHNOLOGY LABORATORIES NAVAL ORDNANCE TEST STATION 


\section{Pacific Journal of Mathematics}

\section{Vol. 12, No. $1 \quad$ January, 1962}

Jonathan L. Alperin, Groups with finitely many automorphisms $\ldots \ldots \ldots \ldots \ldots \ldots \ldots \ldots$

Martin Arthur Arkowitz, The generalized Whitehead product ................ 7

John D. Baum, Instability and asymptoticity in toplogical dynamics . . . . . . . . . . 25

William Aaron Beyer, Hausdorff dimension of level sets of some Rademacher series .... $\quad 35$

Frank Herbert Brownell, III, A note on Cook's wave-matrix theorem . . . . . . . . . . . . . 47

Gulbank D. Chakerian, An inequality for closed space curves ................. 53

Inge Futtrup Christensen, Some further extensions of a theorem of Marcinkiewicz ....... 59

Charles Vernon Coffman, Linear differential equations on cones in Banach spaces . . . . . 69

Eckford Cohen, Arithmetical notes. III. Certain equally distributed sets of integers . . . . . 77

John Irving Derr and Angus E. Taylor, Operators of meromorphic type with multiple poles

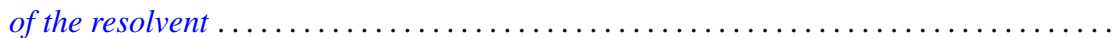

Jacob Feldman, On measurability of stochastic processes in products space .............

Robert S. Freeman, Closed extensions of the Laplace operator determined by a general class of boundary conditions, for unbounded regions ......................

Robert E. Fullerton, Geometric structure of absolute basis systems in a linear topological

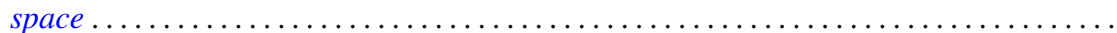

Dieter Gaier, On conformal mapping of nearly circular regions

Andrew Mattei Gleason and Hassler Whitney, The extension of linear functionals defined

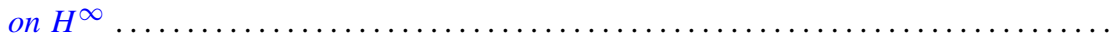

Seymour Goldberg, Closed linear operators and associated continuous linear

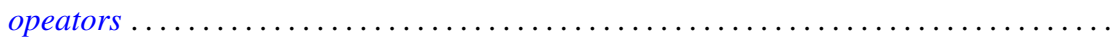

Basil Gordon, Aviezri Siegmund Fraenkel and Ernst Gabor Straus, On the determination of sets by the sets of sums of a certain order

Branko Grünbaum, The dimension of intersections of convex sets. .

Paul Daniel Hill, On the number of pure subgroups

Robert Peter Holten, Generalized Goursat problem . .

Alfred Horn, Eigenvalues of sums of Hermitian matrices ...........

Henry C. Howard, Oscillation and nonoscillation criteria for

$$
y^{\prime \prime}(x)+f(y(x)) p(x)=0
$$

Taqdir Husain, $S$-spaces and the open mapping theorem ...

Richard Eugene Isaac, Markov processes and unique stationary probability measures ...

John Rolfe Isbell, Supercomplete spaces ....................

John Rolfe Isbell, On finite-dimensional uniform spaces. II .........

N. Jacobson, A note on automorphisms of Lie algebras ..............

Antoni A. Kosinski, A theorem on families of acyclic sets and its applications

Marvin David Marcus and H. Minc, The invariance of symmetric functions of singular values...

Ralph David McWilliams, A note on weak sequential convergence.

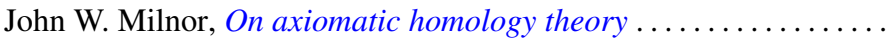

Victor Julius Mizel and Malempati Madhusudana Rao, Nonsymmetric projections in

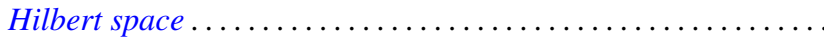

Calvin Cooper Moore, On the Frobenius reciprocity theorem for locally compact

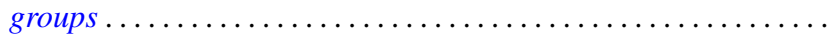

Donald J. Newman, The Gibbs phenomenon for Hausdorff means . 